\title{
Mitochondrial tRNA methylation in Alzheimer's disease and progressive supranuclear palsy
}

Talisa K. Silzer, Gita A. Pathak and Nicole R. Phillips*

\begin{abstract}
Background: Methylation of mitochondrial tRNAs (mt-tRNA) at the 9th position ("p9 site") is known to impact translational efficiency and downstream mitochondrial function; however, direct assessment of mt-RNA methylation is challenging. Recent RNA sequence-based methods have been developed to reliably identify post-transcriptional methylation. Though p9 methylation has been studied in healthy human populations and in the context of cancer, it has not yet been analyzed in neurodegenerative disease, where mitochondrial dysfunction is a prominent and early hallmark of disease progression.
\end{abstract}

Methods: Mitochondrial p9 methylation was inferred from multi-allelic calls in RNA-seq data. Gene-based association studies were performed in FUMA. Correlations between nuclear gene expression and p9 methylation were tested using Spearman's rho. Fisher's Exact test was used in PANTHER and IPA to test for overrepresentation and enrichment of biological processes and pathways in the top nuclear genes correlated with p9 methylation.

Results: Variable methylation was observed at 11 p9 sites in post-mortem cerebellar tissue of elderly subjects who were either healthy or diagnosed with Alzheimer's disease (AD), progressive supranuclear palsy (PSP) or pathological aging (PA). Similarities in degree of methylation were observed between AD and PSP. Certain nuclear encoded genes were identified as significantly associated with p9 methylation. Expression of 5300 nuclear encoded genes was significantly correlated with p9 methylation, with AD and PSP subjects exhibiting similar expression profiles. Overrepresentation and enrichment testing using the top transcripts revealed enrichment for a number of molecular processes, terms and pathways including many of which that were mitochondrial-related.

Conclusion: With mitochondrial dysfunction being an established hallmark of neurodegenerative disease pathophysiology, this work sheds light on the potential molecular underpinnings of this dysfunction. Here we show overlap in cerebellar pathophysiology between common tauopathies such as Alzheimer's disease and progressive supranuclear palsy. Whether $\mathrm{p} 9$ hypermethylation is a cause or consequence of pathology remains an area of focus.

Keywords: Mitochondria, Methylation, Neurodegenerative disease, tRNA

\footnotetext{
* Correspondence: Nicole.Phillips@unthsc.edu

Department of Microbiology, Immunology and Genetics; Graduate School of Biomedical Science, University of North Texas Health Science Center, 3500

Camp Bowie Blvd., Fort Worth, TX, USA
}

C C The Author(s). 2020 Open Access This article is licensed under a Creative Commons Attribution 4.0 International License, which permits use, sharing, adaptation, distribution and reproduction in any medium or format, as long as you give appropriate credit to the original author(s) and the source, provide a link to the Creative Commons licence, and indicate if changes were made. The images or other third party material in this article are included in the article's Creative Commons licence, unless indicated otherwise in a credit line to the material. If material is not included in the article's Creative Commons licence and your intended use is not permitted by statutory regulation or exceeds the permitted use, you will need to obtain permission directly from the copyright holder. To view a copy of this licence, visit http://creativecommons.org/licenses/by/4.0/ The Creative Commons Public Domain Dedication waiver (http://creativecommons.org/publicdomain/zero/1.0/) applies to the data made available in this article, unless otherwise stated in a credit line to the data. 


\section{Background}

Mitochondrial organelles play essential roles in oxidative phosphorylation, apoptosis, calcium homeostasis and a number of cell-signalling pathways. Many lines of evidence have implicated mitochondrial dysfunction in aging and neurodegenerative disease. Though the genetic etiology of neurodegeneration is highly heterogeneous, there is an extensive literature implicating mitochondrial dysfunction as a common and early factor in the pathophysiology of a number of neurodegenerative diseases [1]. Much of the mitochondrial geneticcentric studies of neurodegenerative diseases and related comorbidities have focused primarily on mitochondrial DNA (mtDNA) mutations, changes to mtDNA copy number and/or release of cell-free mtDNA [2]; however, the significance of mitochondrial transcriptome epigenetics and altered mitochondrial RNA (mt-RNA) processing has not been explored. As post-transcriptional processing can alter mitochondrial protein synthesis, aberrant modifications to mitochondrial tRNAs (mt-tRNA) may contribute to the mitochondrial dysfunction underlying neurodegeneration and other age-related pathologies.

Mitochondria contain their own 16,569 base pair genome consisting of 13 mRNA genes encoding electron transport chain subunits and 2 rRNA genes, which are 'punctuated' by 22 tRNA genes [3]. The interspersed tRNA sequences serve as important sites for posttranscriptional processing of the polycistronic transcript, accomplished in part by key modifications at several sites throughout the tRNA structure [4]. One of the most common modification types is methylation of adenosine or guanosine residues [4]. Of particular interest is methylation occurring at the 9th position (termed as "p9 sites" here) of mt-tRNAs, as this modification is known to be essential for proper tRNA folding, stability, and decoding capacity [5-8]. Within mt-tRNAs, methyl groups are added by mitochondrial ribonuclease $\mathrm{P}$ protein 1 (MRPP1) to adenosine to create 1-methyladenosine (m1A). Lack of modification at this position has been associated with rare neurodegenerative disorders such as HSD10 disease [9]. Further, knockdown of key processing molecules such as MRPP1 has been shown to lead to accumulation of improperly processed tRNAs resulting in decreased mitochondrial protein synthesis [10].

Though various methods exist for measuring RNA methylation including in situ hybridization, methylationspecific antibodies and methylation-sensitive enzymes, many methods lack specificity and/or have limited experimental use, due to the high concentration of input RNA required [11]. Several sequence-based protocols have been established and validated demonstrating a strong correlation between variation in mt-RNA sequence data and degree of post-transcriptional methylation of mt-tRNA at specific sites [10, 12, 13]. In brief, the extent of multiallelic calls (seemingly heteroplasmy) within mt-RNA sequence reads can be used to infer degree of post-transcriptional methylation based on the assumption that chemical modifications such as methyl groups, block reverse transcriptase during cDNA synthesis, often leading to incorporation of incorrect nucleotides [14]. Though useful for detecting methylation, it is important to note that this method likely leads to the underestimation of methylation rate.

Using RNA sequence-based approaches, previous groups have identified variable methylation across 11 different $\mathrm{p} 9$ sites within mt-tRNAs of healthy Caucasian individuals; this methylation was discovered to be significantly associated with nuclear gene variants (i.e. MRPP3) [15]. In this study, we examined methylation at these same 11 mitochondrial $\mathrm{p} 9$ sites in the context of different neurodegenerative pathologies and identified associated nuclear genetic variants and gene expression. The results point to altered cellular processes that may be contributing to the mitochondrial dysfunction occurring within the cerebellum that is a hallmark of both Alzheimer's disease (AD) and progressive supranuclear palsy (PSP).

\section{Methods}

\section{Samples used}

Samples used for this study were post-mortem cerebellar tissue of 266 elderly Caucasian individuals who were either healthy or diagnosed with Alzheimer's disease (AD), progressive supranuclear palsy (PSP) or pathological aging (PA) (Table 1). Samples were originally collected by the Mayo RNAseq study led by Dr. Nilüfer ErtekinTaner, Mayo Clinic, Jacksonville, FL as part of the multiPI U01 AG046139 (MPIs Golde, Ertekin-Taner, Younkin, Price). All individuals with AD “...had definite diagnosis according to the NINCDS-ADRDA criteria and had Braak NFT stage of IV or greater. Control subjects had Braak NFT stage of III or less, CERAD neuritic and cortical plaque densities of 0 (none) or 1 (sparse) and lacked any of the following pathologic diagnoses: $\mathrm{AD}$, Parkinson's disease (PD), DLB, VaD, PSP, motor neuron disease (MND), CBD, Pick's disease (PiD), Huntington's disease (HD), FTLD, hippocampal sclerosis (HipScl) or dementia lacking distinctive histology (DLDH)" (doi: https://doi.org/10.7303/syn5550404).

\section{Data obtainment and QC}

SNP, RNA-seq data and gene expression data were obtained from the Synapse Data Repository (doi:https:// doi.org/10.7303/syn5550404). Genomic and RNA-seq data was originally generated and QC'd by the Golde, Ertekin-Taner, Younkin and Price laboratories. Briefly, genotypes were obtained using the Illumina Human 
Table 1 Demographics of subjects used

\begin{tabular}{llllll}
\hline Diagnosis & Sex & Age at & APOE & \\
\cline { 5 - 6 } & & Death & e2 & e3 & e4 \\
\hline NC $(n=74)$ & Male $(n=40)$ & $80.93 \pm 8.15$ & $7.43 \%$ & $86.40 \%$ & $6.08 \%$ \\
& Female $(n=34)$ & $85.15 \pm 6.76$ & & & \\
AD $(n=82)$ & Male $(n=34)$ & $81.68 \pm 8.17$ & $2.44 \%$ & $68.30 \%$ & $29.30 \%$ \\
& Female $(n=48)$ & $83.21 \pm 7.19$ & & & \\
PSP $(n=83)$ & Male $(n=51)$ & $74.22 \pm 6.94$ & $9.04 \%$ & $82.53 \%$ & $8.43 \%$ \\
& Female $(n=32)$ & $73.65 \pm 5.99$ & & & \\
PA $(n=27)$ & Male $(n=11)$ & $85.18 \pm 3.74$ & $7.41 \%$ & $75.92 \%$ & $16.67 \%$ \\
& Female $(n=16)$ & $84.06 \pm 4.65$ & & & \\
\hline
\end{tabular}

Age is reported as an average \pm standard deviation for each respective group. Normal control (NC), Alzheimer's disease (AD), progressive supranuclear palsy (PSP), pathological aging (PA).

Omni 2.5Exome array. The protocol used for QC of genomic data included the following procedures: (1) duplicate or relatedness, (2) discrepant sex information, (3) low genotyping call rate (98\%) and (4) outlying heterozygosity ( \pm 3 standard deviations from the mean) were removed using PLINK. EIGENSTRAT was used to identify outliers ( $>6$ standard deviations from the mean after 5 iterations) [16]. Based on this QC protocol, a total of 10 individuals were removed from the original dataset of 278 individuals.

Briefly, RNA was extracted from brain tissue using TRIzol $^{\circ}$ and Qiagen RNeasy columns treated with DNase. Sequencing libraries were prepared using the Illumina Truseq kit. An Illumina Hiseq 2000 was used to obtain $101 \mathrm{bp}$ paired-end reads (performed in triplicate; 3 lanes/sample). RNA-seq data of individuals with low mapped reads $(<85 \%)$ or sex discrepant gene counts (Ychromosome gene expression) were removed from further analysis (20 individuals total). Using SNAPR, raw RNAseq reads were aligned using the human genome and transcriptome builds GRCh38 and GRCh38.77 respectively, while filtering on Phred scores $<=20$ [17]. Subsequent BAM files were utilized for determining alternative allele frequency at the $11 \mathrm{p} 9$ sites. Gene expression was derived from transcript counts generated using SNAPR [17]; counts were normalized using the trimmed mean of $\mathrm{m}$ values (TMM) method in edgeR taking into account differences in library size (calcNormFactors).

\section{Mitochondrial RNA heteroplasmy}

The aim of this study was to investigate posttranscriptional mitochondrial p9 methylation in the context of neurodegenerative phenotypes. Mitochondrial specific reads were extracted from total RNA-seq BAM files using samtools v.1.34 view. An mpileup file was created from each BAM file using samtools mpileup. Heteroplasmy was measured at 11 different $\mathrm{p} 9$ sites in the mitochondrial transcriptome $(585,1610,4271,5520$,
7526, 8303, 9999, 10,413, 12,146, 12,274, 14,734) from the mpileup files using readcounts in VarScan v.2.4 with default parameters. Of note, none of the aforementioned p9 sites have previously been shown to overlap with mitochondrial pseudogene (i.e. nuclear mitochondrial DNA (NUMT)) sequences [15]. Frequency of alternative allele calls (non-reference calls based on Cambridge Reference Sequence (CRS)) was measured at each of the 11 sites. Degree of post-transcriptional methylation was inferred from the frequency of alternative allele calls based on previous published methods [15]. A KolmogorovSmirnov test determined the data to have a non-normal distribution. Correlation coefficients for $\mathrm{p} 9$ methylation across all 11 sites were determined using Spearman's rho. Degree of p9 methylation was analyzed between disease states using pairwise Kruskal-Wallis tests with Bonferroni correction for multiple testing at each individual site (not accounting for the 11 site tests).

\section{Gene-based genome-wide association}

Gene-based association studies were utilized to identify nuclear encoded genes significantly associated with p9 methylation. In brief, principle component analysis using EIGENSOFT was conducted to determine if a considerable amount of heterogeneity was present in the SNP data [18]. Linkage disequilibrium-based pruning of the SNPs was conducted in PLINK (--indep-pairwise 5050.2 ) yielding a final dataset of 58,174 SNPs in linkage equilibrium [19]. Linear association adjusting for age, sex and eigenvectors 1-10 was applied to identify genetic variants associated with degree of methylation ( $\log 10$ transformed) at each respective p9 site using the SNP2GENE function of the Functional Mapping and Annotation (FUMA) platform [20]; this utilizes MAGMA to map SNPs to protein-coding genes [21]. Position map window size was conservatively set to 10 $\mathrm{kb}$. The genome-wide significance threshold was set to a Bonferroni adjusted $p$-value of $2.93 \times 10^{-6}$ based on the mapping of input SNPs to 17,079 genes; Bonferroni correction was applied within each linear association test. Manhattan plots and local regional plots as well as Q-Q plots to identify genomic inflation in the SNP-set used for the gene-based GWAS, were generated in FUMA. CADD scores reported for each gene are based on the top leading $\mathrm{SNP} /$ posMapMaxCADD as determined in FUMA.

\section{Gene expression analysis}

To identify potential downstream effects (i.e. biological changes) linked to $\mathrm{p} 9$ methylation, we investigated nuclear gene expression significantly correlated with methylation. Correlations between nuclear genes and methylation at site 585 were determined using Spearman's rho. The top 1000 transcripts (565 unique genes) 
and the first two principle components were visualized using the ClustVis online tool [22]. Rows and columns of the heatmap were clustered based on correlation distance and average linkage with unit variance scaling. The top genes (based on top 1000 transcripts) were input into the PANTHER classification system to identify overrepresented cellular components, molecular functions and/or biological processes using Fisher's exact test with FDR correction [23]. For a more targeted enrichment analysis, all 14,306 transcripts (5300 unique genes) significantly correlated with $\mathrm{p} 9$ methylation were input into Ingenuity Pathway Analysis (IPA) (Qiagen Inc., https://www.qiagenbioinformatics.com/products/ingenuitypathway-analysis) and were tested for pathway enrichment using the Core Analysis function and a cerebellum specific reference.

\section{Results}

\section{Mitochondrial tRNA methylation}

Frequencies of alternative allele calls measured at 11 previously identified p9 sites $(585,1610,4271,5520$, 7526, 8303, 9999, 10,413, 12,146, 12,274, 14,734) based on the revised Cambridge reference sequence, were used to infer degree of post-transcriptional methylation. High levels of mt-RNA sequence variation were observed across all individuals (Additional Figure 1). Degree of methylation, inferred from multi-allelic calls in RNA-seq data, was highly correlated across all $11 \mathrm{p} 9$ sites with exception of 4271 and 14,734 (Additional Figure 2). For each $\mathrm{p} 9$ site, comparisons were made between each of the four groups: normal controls (NC) $(n=74)$, Alzheimer's disease $(\mathrm{AD})(n=82)$, progressive supranuclear palsy (PSP) $(n=83)$ and pathological aging (PA) $(n=27)$ to assess differences in methylation levels. Nonparametric (Kruskal-Wallis) pairwise comparisons by disease state revealed significant differences in methylation at a number of $\mathrm{p} 9$ sites, with AD and PSP showing similar degrees of hypermethylation in comparison to $\mathrm{NC}$ and PA; no significant difference was seen between $\mathrm{NC}$ and PA (Table 2; Additional file 4). Site 585 was found to be the most variable, though the reasoning for this is unclear.

\section{Nuclear gene-based associations}

Gene-based GWAS mapped typed SNPs to 17,079 protein coding genes. Two SNPs (rs12343928; rs4877837) located on chromosome 9 were mapped to SLC28A3 (pvalue $=1.935 \times 10^{-6}$, CADD score $\left.=24.7\right)$ and were determined to be significantly associated with methylation at site 585; the regional plot reveals that several SNPs in LD with the top lead SNP are also associated in the local signal (Fig. 1; Additional file 2). A single SNP (rs2034879) mapping to SENP8 (CADD score $=13.22$ ), MYO9A $\quad($ CADD score $=18.35), \quad$ GRAMD2 $\quad($ CADD score $=16.49)$ on chromosome 15 had suggestive associations with methylation at p9 site 585 (Fig. 1; Additional file 2). Another SNP (rs9872864) mapping to TRAIP ( $p$ value $=8.32 \times 10^{-7}$, CADD score $\left.=7.558\right)$ and nearby gene IP6K1 $\left(\mathrm{p}\right.$-value $=6.67 \times 10^{-7}$, CADD score $\left.=20.7\right)$ located on chromosome 3 was also found to have significant associations with methylation at six out of the 11 p9 sites (5520, 7526, 8303, 9999, 10,413, 12,146) (Fig. 2; Additional file 2).

\section{Mitochondrial-related gene expression}

Expression of 5300 transcripts was found to significantly correlate with degree of methylation at site 585; the top 1000 transcripts (565 unique genes) are visualized in Fig. 3. Based on the primary bifurcation, left and right clades appear to be enriched for AD and PSP, and NC and PA respectively; though enrichment was not formally tested (Fig. 3).

The top 1000 transcripts correlated with p9 methylation at site 585 were analyzed using PANTHER to test for significantly overrepresented cellular components, molecular functions and biological processes. We identified a number of GO terms to be overrepresented in our gene-set including several that were mitochondriallyassociated (Fig. 4; Additional file 3).

A tissue-specific (i.e. cerebellum) enrichment analysis was then conducted in IPA using all significantly correlated transcripts (5300 unique genes). Several pathways were identified as enriched in our gene-set including many that are linked to mitochondrial function as well as DNA damage/repair (Fig. 5).

\section{Discussion}

Our analysis of RNA sequence has validated that methylation occurs consistently at functionally important sites across the mitochondrial transcriptome, and that this methylation is highly correlated across the 11 multiallelic sites. Here we show significant hypermethylation at specific sites in the mitochondrial transcriptome (i.e. mttRNA p9 sites) in the cerebellar tissue of individuals with Alzheimer's disease and progressive supranuclear palsy. Similarities in methylation between these two pathologies may suggest a comparable genetic etiology underlying the cerebellar mitochondrial dysfunction that is a hallmark of both diseases [24], [25]. Interestingly, no significant difference was observed between NC and PA, which may be due to the ambiguity of differentiating healthy from pathological aging. Individuals diagnosed with pathological aging often appear to be cognitively intact despite the presence of neuritic plaques. Similar presentation has also been observed in healthy aged individuals, making it difficult to distinguish between the two diagnoses [26]. Moreover, a recent study by Idaghdour and Hodgkinson (2017) revealed significant 
Table 2 Summary table detailing p9 methylation data distributions and significant group differences per site

\begin{tabular}{|c|c|c|c|c|c|c|}
\hline p9 site & & NC & AD & PSP & PA & \\
\hline \multirow{5}{*}{585} & Mean \pm SD & $0.0140 \pm 0.0057$ & $0.0210 \pm 0.01067$ & $0.0201 \pm 0.0092$ & $0.0142 \pm 0.0053$ & p-value (Bonferroni adj.) \\
\hline & NC & & $<0.001$ & $<0.001$ & & \multirow{4}{*}{$<0.001$} \\
\hline & $A D$ & $* *$ & & & 0.01 & \\
\hline & PSP & $* *$ & & & 0.02 & \\
\hline & PA & & * & * & & \\
\hline \multirow{5}{*}{1610} & & $0.1276 \pm 0.0456$ & $0.1601 \pm 0.0579$ & $0.1546 \pm 0.0599$ & $0.1239 \pm 0.0339$ & \multirow{5}{*}{0.001} \\
\hline & NC & & 0.003 & 0.045 & & \\
\hline & $A D$ & * & & & 0.018 & \\
\hline & PSP & * & & & 0.1 & \\
\hline & PA & & $*$ & & & \\
\hline \multirow[b]{2}{*}{4271} & & $0.1308 \pm 0.0247$ & $0.1343 \pm 0.0234$ & $0.1304 \pm 0.0229$ & $0.1379 \pm 0.0239$ & \multirow[b]{2}{*}{0.602} \\
\hline & \multicolumn{5}{|c|}{ No significant group differences } & \\
\hline \multirow{5}{*}{5520} & & $0.0610 \pm 0.0393$ & $0.0810 \pm 0.0409$ & $0.0790 \pm 0.0496$ & $0.0576 \pm 0.0250$ & \multirow{5}{*}{0.002} \\
\hline & NC & & 0.004 & 0.072 & & \\
\hline & $A D$ & * & & & 0.069 & \\
\hline & PSP & & & & 0.357 & \\
\hline & PA & & & & & \\
\hline \multirow{5}{*}{7526} & & $0.0954 \pm 0.0468$ & $0.1217 \pm 0.0492$ & $0.1247 \pm 0.0685$ & $0.0888 \pm 0.0353$ & \multirow{5}{*}{0.001} \\
\hline & NC & & 0.007 & 0.059 & & \\
\hline & $A D$ & * & & & 0.019 & \\
\hline & PSP & & & & 0.83 & \\
\hline & PA & & $*$ & & & \\
\hline \multirow{5}{*}{8303} & & $0.0687 \pm 0.0419$ & $0.0830 \pm 0.0455$ & $0.0966 \pm 0.0572$ & $0.0758 \pm 0.0346$ & \multirow{5}{*}{0.01} \\
\hline & NC & & 0.17 & 0.006 & & \\
\hline & $A D$ & & & & & \\
\hline & PSP & * & & & & \\
\hline & PA & & & & & \\
\hline \multirow{5}{*}{9999} & & $0.0929 \pm 0.0527$ & $0.1130 \pm 0.0546$ & $0.1370 \pm 0.0808$ & $0.0921 \pm 0.0416$ & \multirow{5}{*}{0.001} \\
\hline & NC & & 0.072 & 0.001 & & \\
\hline & $A D$ & & & & 0.518 & \\
\hline & PSP & $* *$ & & & 0.055 & \\
\hline & PA & & & & & \\
\hline \multirow{5}{*}{10413} & & $0.0754 \pm 0.04986$ & $0.1000 \pm 0.0514$ & $0.1209 \pm 0.0857$ & $0.0728 \pm 0.0389$ & \multirow{5}{*}{$<0.001$} \\
\hline & NC & & 0.007 & $<0.001$ & & \\
\hline & $A D$ & * & & & 0.131 & \\
\hline & PSP & $* *$ & & & 0.031 & \\
\hline & PA & & & $*$ & & \\
\hline \multirow[b]{2}{*}{12146} & & $0.0445 \pm 0.0278$ & $0.0523 \pm 0.0289$ & $0.0658 \pm 0.0512$ & $0.0492 \pm 0.0232$ & \multirow[b]{2}{*}{0.063} \\
\hline & \multicolumn{5}{|c|}{ No significant group differences } & \\
\hline \multirow{5}{*}{12274} & & $0.0103 \pm 0.0069$ & $0.0140 \pm 0.0086$ & $0.0167 \pm 0.0151$ & $0.0099 \pm 0.0059$ & \multirow{5}{*}{0.004} \\
\hline & NC & & 0.038 & 0.024 & & \\
\hline & $A D$ & * & & & 0.22 & \\
\hline & PSP & * & & & 0.17 & \\
\hline & PA & & & & & \\
\hline & & $0.0480 \pm 0.0211$ & $0.0484 \pm 0.0480$ & $0.0474 \pm 0.0226$ & $0.0450 \pm 0.0178$ & \\
\hline 14734 & & Nos & ignificant group dif & fferences & & 0.933 \\
\hline
\end{tabular}

Mean \pm standard deviation of degree of methylation (inferred from alternative allele frequency) is shown across the top for each site. Non-parametric KruskalWallis $p$-values with Bonferroni correction (to adjust for multiple comparisons) is shown on the far right. $P$-values from pair-wise Kruskal-Wallis comparisons between groups are shown within the matrix; p-values $<0.05(*)$, p-values $\left.<0.001{ }^{* *}\right)$.

hypermethylation at mitochondrial $\mathrm{p} 9$ sites in cancer. This substantiates the notion that mt-tRNA hypermethylation may be more closely associated with pathology rather than normal aging processes. Whether this hypermethylation is a cause or consequence of pathology is still unclear.
Nevertheless, it is important to acknowledge the technical limitations associated with traditional RNA sequencing workflows. Here we sought to investigate posttranscriptional methylation of mt-tRNAs using total RNA sequence data. Importantly, these RNA molecules are highly folded and small in nature, often making them 


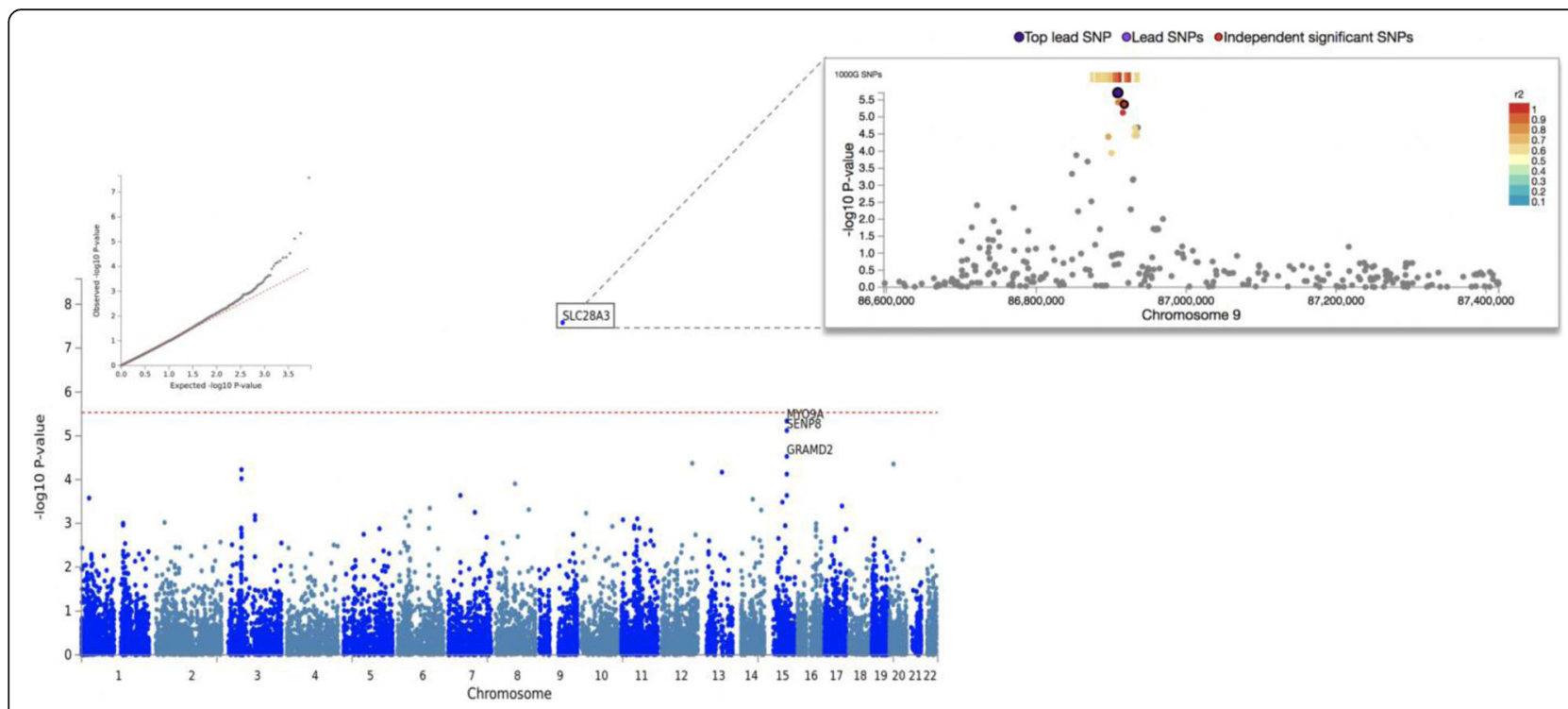

Fig. 1 Association results for gene-based GWAS with methylation at site 585. The Q-Q plot (top left) conforms to expectations and shows no sign of genomic inflation. The Manhattan plot (bottom left) illustrates a primary signal in SLC28A3 on chromosome $9\left(p-v a l u e=1.935 \times 10^{-6}\right)$; as well as multiple suggestive associations on chromosome 15. The regional plot (top right) displays clustering of individual SNPs within an $800 \mathrm{~kb}$ window and shows multiple SNPs in LD with the top lead SNP in the gene-based signal

difficult to capture. Traditional total RNA sequencing protocols, such as that used here often 1) do not enrich for, and 2) usually detail removal of, small RNAs. This means that mature mt-tRNA reads may have been under-represented in this dataset. Therefore, any conclusions made here may not be based on the entire mature mt-tRNA pool. Future studies investigating p9 methylation should aim to use small RNA enrichment protocols, as well as perform cDNA size selection prior to RNA sequencing.

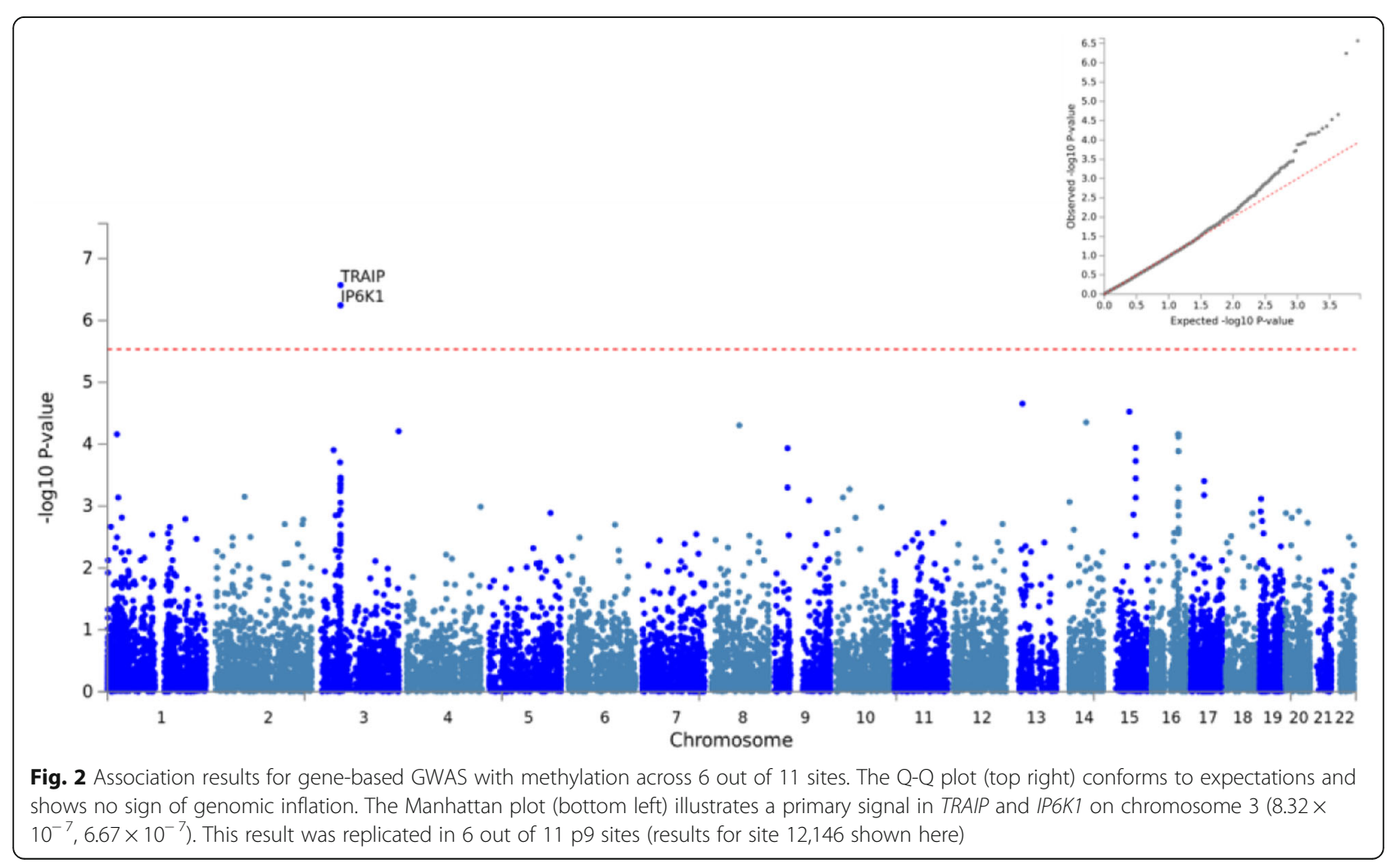




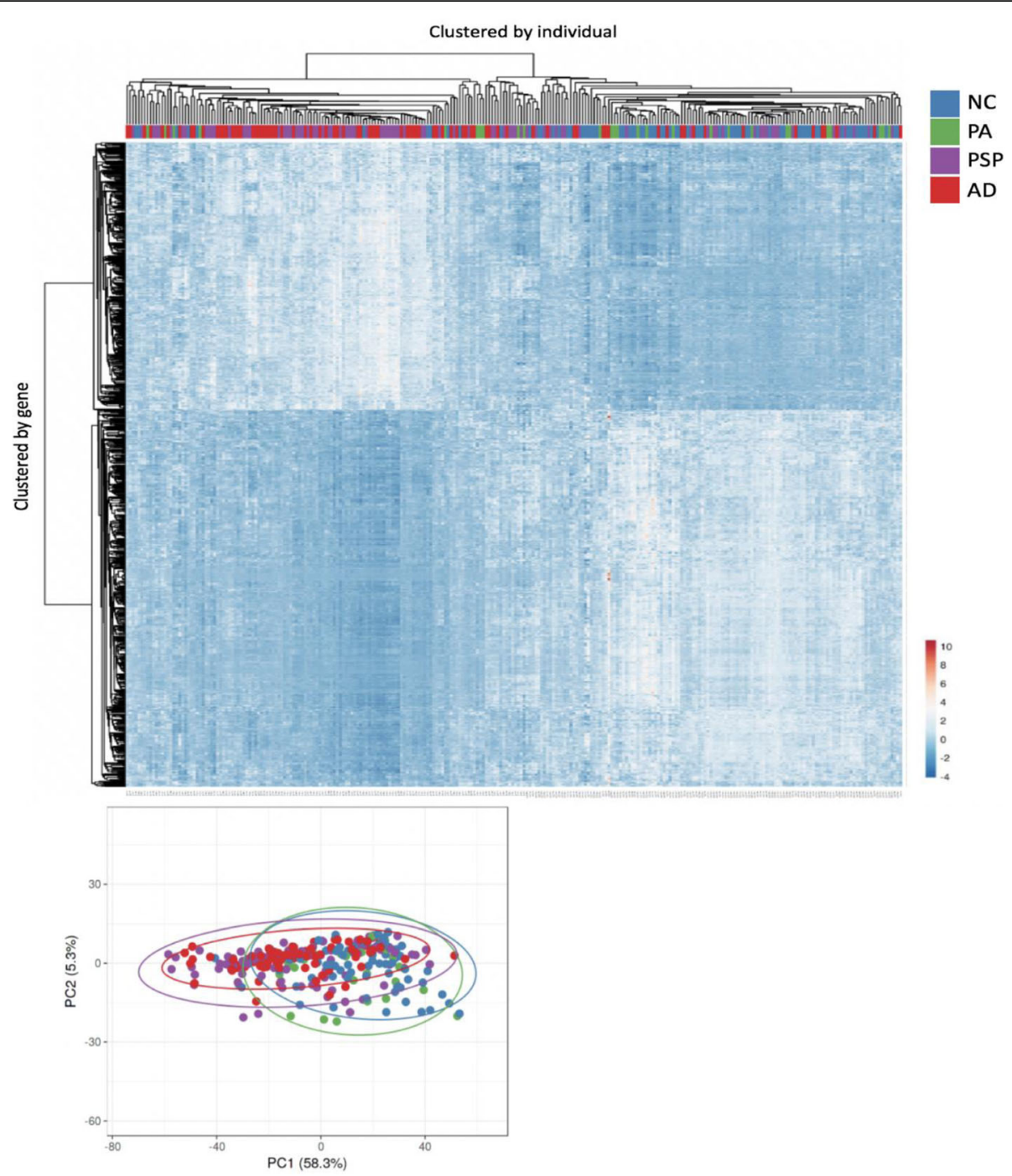

Fig. 3 Heatmap displaying the top 1000 transcripts significantly associated with degree of p9 methylation at site 585. Expression profiles are clustered by gene (y-axis) and individual (x-axis) (top). Principle component analysis of gene expression displays overlap between case groups (bottom left). Colors represent disease status; NC (blue), PA (green), AD (red) and PSP (purple) (bottom). Gradient bar (bottom right) represents gene expression level; high expression (red), low expression (blue)

\section{Nuclear genes are associated with p9 methylation}

The gene-based genome-wide association analysis revealed that variation in several nuclear encoded genes is significantly associated with methylation at different $\mathrm{p} 9$ sites throughout the mitochondrial transcriptome. Two SNPs (rs12343928; rs4877837) mapping to solute carrier family 28 member 3 (SLC28A3) located on chromosome 9 were found to be significantly associated with methylation at $\mathrm{p} 9$ site 585 . The strength of this result was further substantiated by the clustering of neighbouring SNPs in linkage disequilibrium within the gene region (Fig. 1). The protein encoded by this gene is involved in regulating neurotransmission, vascular tone and metabolism of nucleoside drugs, which are processes that are physiologically relevant to our tissue and diseases of interest. Nucleoside drugs are often used to treat agerelated diseases such as type 2 diabetes and hypertension, as well as mitochondrial dysfunction in Parkinson's disease (PD) [27]. This is interesting, as PSP pathology also features mitochondrial dysfunction and bears phenotypic similarity to $\mathrm{PD}$, while $\mathrm{T} 2 \mathrm{D}$ and hypertension often serve as comorbidities for AD.

A single SNP (rs2034879) that mapped to several genes (SENP8, MYO9A, GRAMD2) on chromosome 15 (likely due to the restrictive window size of $\pm 10 \mathrm{~kb}$ that was used) reached the suggestive threshold for association with p9 methylation at position 585. SENP8 is known to catalyze pathways associated with neddylation, a post-transcriptional modification analogous to ubiquitination; these pathways are also responsible for the 


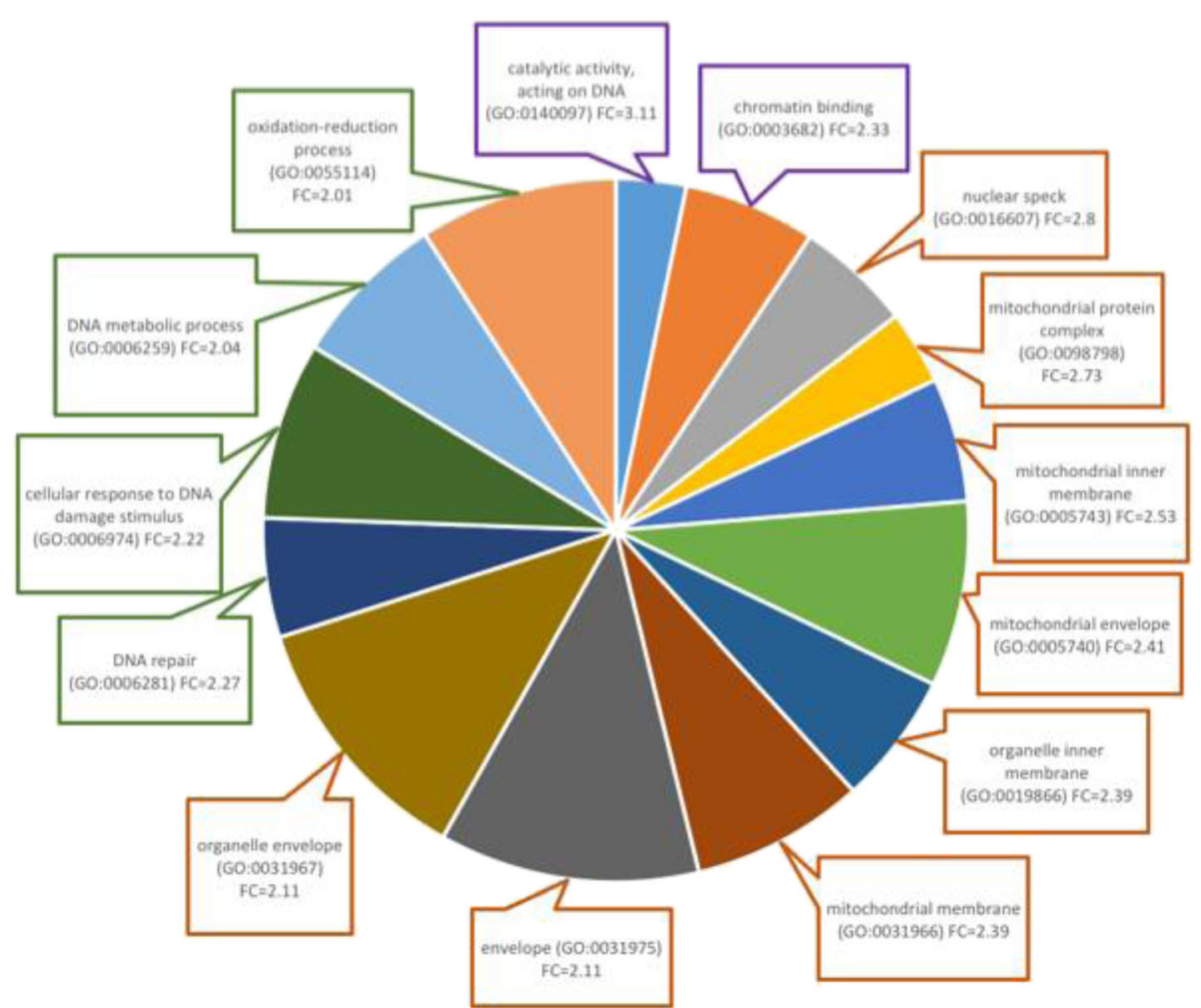

Fig. 4 Overrepresented GO terms based on the top 1000 transcripts significantly correlated with p9 methylation. GO terms are color coded by category; Molecular function (purple), cellular component (orange), biological process (green). Terms were selected using a threshold of $>2$ fold change. Fold change was determined based on number of enriched genes from our 1000 transcripts list divided by the number of expected enriched genes

endocytic degradation of APP. Dysfunction of these pathways has been observed in AD leading to characteristic accumulation of APP and A $\beta$ [28]. MYO9A is an unconventional myosin responsible for the regulation of Rho GTPase activity within neurons, which in turn regulates neuronal morphology and function; for these reasons, the Rho family has been a recent therapeutic target for neurodegenerative diseases such as AD [29]. In turn, various Rho GTPases have also been implicated in maintenance of mitochondrial homeostasis and

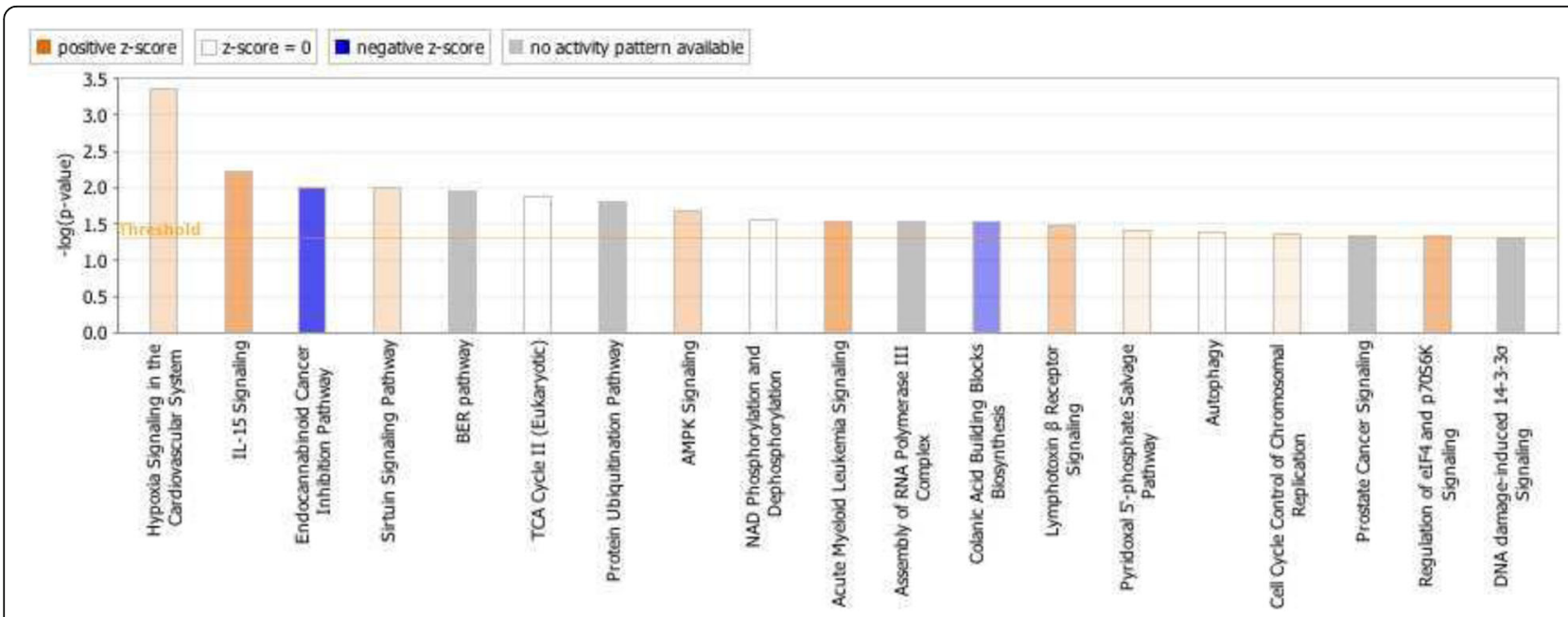

Fig. 5 Enriched canonical pathways based on all transcripts significantly correlated with p9 methylation. Pathways positively and negatively correlated with p9 methylation are shown in orange and blue respectively 
apoptotic signaling [30]. GRAMD2 is involved in the organization of endoplasmic reticulum-plasma membrane contact sites (EPCS), which are key modulators of calcium homeostasis [31]. Calcium serves as an important regulator of mitochondrial bioenergetics (e.g. activation of different Kreb's cycle enzymes [32]), dynamics and apoptotic signaling. Subsequent contact sites between the ER and mitochondria, termed mitochondrial associated membranes (MAMs), facilitate uptake of calcium from the ER to the mitochondria [33], although there is not a clear role for GRAMD2 in this process.

Methylation at six of the $11 \mathrm{p} 9$ sites $(5520,7526,8303$, $9999,10,413,12,146)$ was significantly associated with a single SNP (rs9872864) mapping to two genes TRAIP and IP6K1 located on chromosome 3. TRAIP encodes TRAF interacting protein, an E3 ubiquitin ligase that plays a key role in cell survival and apoptosis. TRAIP serves important roles in cell cycle checkpoints by regulating spindle assembly, appropriate chromosome distribution during cell division, and DNA damage responses [34]. Cell cycle checkpoints have been proposed to serve neuroprotective roles; checkpoint dysregulation and cell cycle re-entry of post-mitotic neurons have been observed in a number of tauopathies [35]. IP6K1 encodes an inositol phosphokinase responsible for synthesis of 5diphosphoinositol phentakisphosphate $\left(5-\mathrm{IP}_{7}\right)$ from hexakisphosphate $\left(\mathrm{IP}_{6}\right)$. IP6K1 serves as an important upstream regulator for various metabolic processes (e.g. glucose homeostasis, lipolysis), apoptosis and global transcription [36]. It has been investigated as a therapeutic target for obesity and type 2 diabetes [37] and variants within the IP6K1 gene region have been previously identified, using tag SNP analysis, as being associated with $\mathrm{AD}$ [38]. IP6K1 also impacts mitochondrial function by regulating ATP concentration via alteration of the ratio of glycolytic to oxidative phosphorylation [39]. Knockout (IP6K1-/-) yeast models have shown decreased mitochondrial respiration, yet increases in ATP, a paradox that is thought to be due to enhanced glycolysis and depletion of metabolic processes requiring ATP [39]. Further, its role in lipolysis is pathologically relevant as findings from Wan et al. [40] demonstrate the capacity of $A \beta$ accumulation to promote lipolysis, increasing lipid toxicity and lipid peroxidation, leading to subsequent downstream mitochondrial dysfunction [41], a phenotype common to many neurodegenerative diseases.

While several of the genes associated here are loosely connected to mitochondrial function, we did not identify any loci that may directly affect mt-tRNA processing. It is interesting to note that 5 out of the 6 genes identified in the gene-based GWAS had CADD scores $>10$ (based on the top leading SNP or maximum CADD score of variants within the gene region). In brief, Combined Annotation Dependent Depletion (CADD) scoring is a predictive machine learning tool that allows for estimation of the deleteriousness and/or pathogenicity of causal genetic variants [42]. In general, raw CADD scores exceeding 10 are considered to be in the top $10 \%$ of deleterious variants in the human genome [42]. Our findings here suggest that the genes identified as having significant associations with $\mathrm{p} 9$ methylation, may play a role in pathogenesis; though their exact role remains ambiguous at this time.

Hodkinson et al. [15], reported p9 methylation associations with several genes-one of which is MRPP3, a key player in tRNA processing in the mitochondria. We did not replicate the MRPP3 association which is not entirely surprising since their study was conducted in normal adults (40-69 years) without any particular pathological conditions versus our study of aged subjects with neurodegenerative disease. It may follow that the variability in MRPP3 associated with p9 methylation levels described in Hodgkinson et al., is presumably normal, whereas p9 methylation in our cohort is presumably a result of pathological process(es). The resulting genotype associations may be pointing to novel gene variants that are upstream effectors of mitochondrial function. Conversely, it is important to acknowledge the caveat that gene-based association testing was performed together on all individuals of this cohort; the small sample size of each diagnostic category (NC, AD, PSP, PA) left us underpowered to detect significant associations when analyzing by each group separately. Given that we identified p9 hypermethylation in both AD and PSP, it is possible that genes identified here as significantly associated with $\mathrm{p} 9$ methylation may be related to individual pathologies and not necessarily post-transcriptional RNA processes.

\section{Mitochondrially-related gene expression is correlated with $\mathrm{p} 9$ methylation}

We identified 5300 genes significantly correlated with $\mathrm{p} 9$ methylation. Using principle component analysis, we identified clustering of gene expression profiles by disease state with AD and PSP, and NC and PA grouping together respectively. Of the top gene expression hits, we identified over-represented GO terms related to a number of cellular components and processes using PANTHER. Interestingly, there was over-representation of mitochondrial components.

In order to identify tissue-specific changes, we performed further enrichment analysis in IPA using a cerebellum tissue reference and all of the transcripts $(5300$ unique genes) significantly correlated with p9 methylation. Again, several of the enriched pathways identified were linked to mitochondrial function and homeostasis, as well as DNA damage/repair. We also identified a number of pathways related to RNA processing. In 
addition, some of the GO terms (e.g. DNA repair and cellular response to DNA damage stimulus) and canonical pathways (e.g. Cell cycle control of chromosomal replication, DNA damage induced 14-3-3 $\sigma$ signaling) appeared to correlate with functions of one of the top genes (i.e.TRAIP) identified in the gene-based GWAS. Though some of the GO terms and canonical pathways are rather ambiguous, it does imply that $\mathrm{p} 9$ methylation is associated with altered transcription of gene-sets important for mitochondrial function.

\section{Conclusion}

This is the first work analyzing mitochondrial p9 methylation in the context of neurodegeneration. Here we report an association between $\mathrm{p} 9$ methylation and nuclearencoded gene expression, as well as mitochondrialrelated regulation; although, the implications of these associations are unclear. We observed comparable posttranscriptional mt-tRNA hypermethylation and nuclear gene expression profiles in the cerebellum of Alzheimer's disease and progressive supranuclear palsy patients. Although technical limitations in this analysis do not allow us to conclude on methylation status of the mature tRNA pool, the results here are likely indicative of molecular similarities underlying the mitochondrial dysfunction already known to occur within the cerebellum of individuals diagnosed with either of these tauopathies.

A basal level of p9 methylation is required for proper tRNA folding and stability [7] and the degree of methylation at $\mathrm{p} 9$ sites has been shown by other groups to have low inter-individual variability [43]. In addition, p9 hypermethylation has been observed in other age-related diseases such as cancer [44]. We propose that p9 hypermethylation in AD and PSP may be more closely associated with pathology as opposed to normal aging. As shown in Fig. 6, we observed that specific nuclear encoded variants associated with hypermethylation at mitochondrial p9 sites within cerebellar neurons, importantly causality is not clear (i.e. associations may be linked to pathological processes and not necessarily RNA methylation). For example, hypermethylation of $\mathrm{p} 9$ may be the primary event that impacts the function of the electron transport chain (ETC) and results in mitochondrial dysfunction, due to the influence that p9 methylation has on downstream mitochondrial protein translation. Alternatively, it is possible that ETC dysfunction is the primary event that causes altered p9 methylation, in which case, the p9 hypermethylation is serving as an endophenotype of genetic risk for mitochondrial dysfunction. Regardless, through retrograde signaling, mitochondrial dysfunction may impact nuclear gene expression resulting in alterations in cellular and

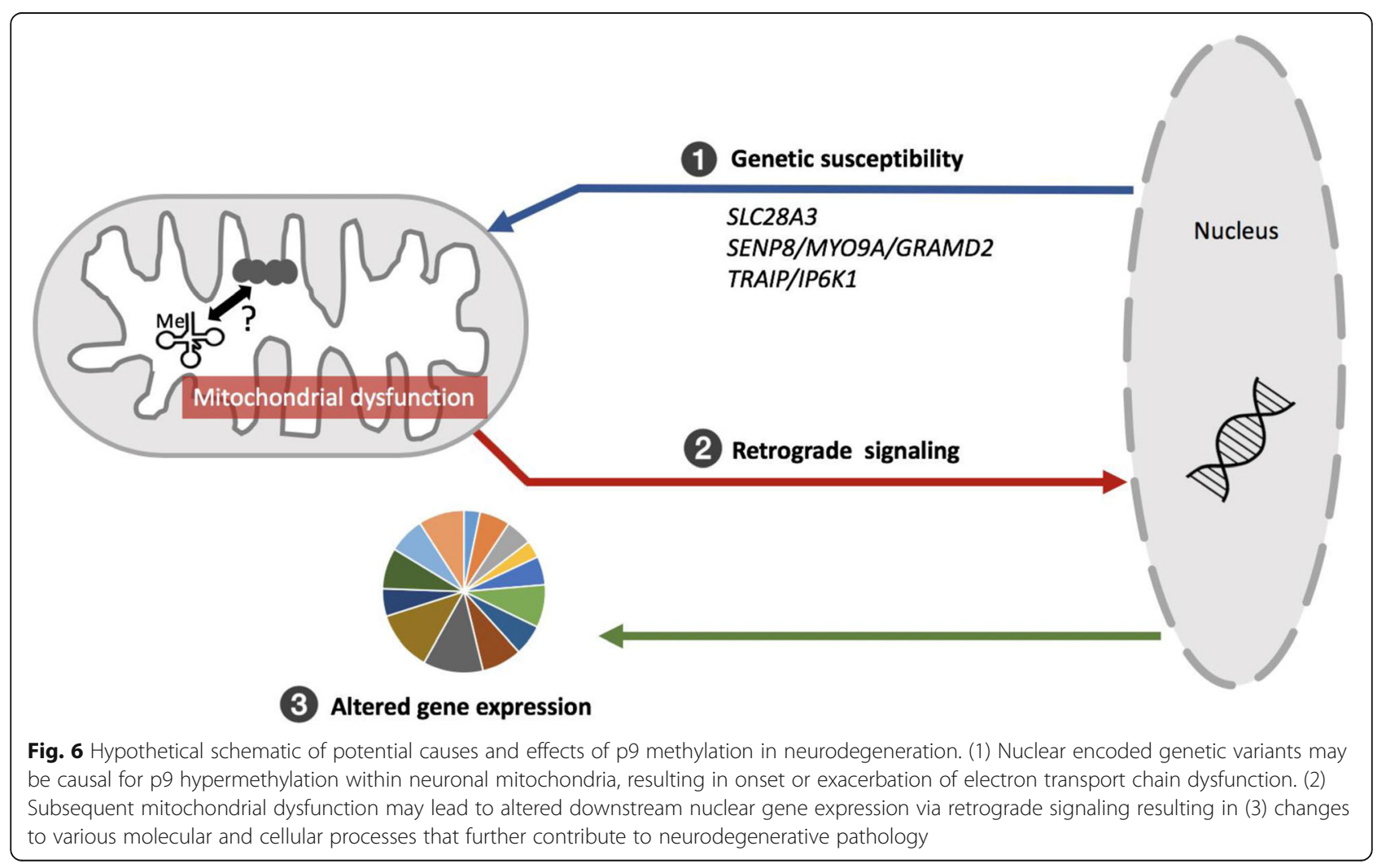


molecular processes to further exacerbate neurodegenerative pathology.

Though at present, it is unclear whether mt-tRNA methylation is a cause or consequence of pathology, these results point to a potential role for mitochondrial posttranscriptional methylation in the pathophysiology of common tauopathies such as Alzheimer's disease and progressive supranuclear palsy. Given this, post-transcriptional mttRNA methylation may serve as an exciting area of investigation for the development of future treatment strategies for neurodegenerative disease.

\section{Supplementary information}

Supplementary information accompanies this paper at https://doi.org/10. 1186/s12920-020-0727-9.

Additional file 1. Additional figures.

Additional file 2. Gene-based GWAS results.

Additional file 3. PANTHER over-representation test results.

Additional file 4. Boxplot by diagnosis.

\section{Abbreviations}

mtDNA: Mitochondrial DNA; mt-RN: Mitochondrial RNA; mttRNA: Mitochondrial tRNA; p9: Position nine; NC: Normal control; AD: Alzheimer's disease; PSP: Progressive supranuclear palsy; PA: Pathological aging; GWA: Genome-wide association study; SNP: Single nucleotide polymorphism; FDR: False discovery rate; CADD: Combined Annotation Dependent Depletion; NUMT: Nuclear mitochondrial DNA; ETC: Electron transport chain; EPCS: Endoplasmic reticulum-plasma membrane contact sites; MAMs: Mitochondrial associated membranes; TMM: Trimmed mean of m-value

\section{Acknowledgements}

Study data were provided by the following sources: The Mayo Clinic Alzheimer's Disease Genetic Studies, led by Dr. Nilufer Taner and Dr. Steven G. Younkin, Mayo Clinic, Jacksonville, FL using samples from the Mayo Clinic Study of Aging, the Mayo Clinic Alzheimer's Disease Research Center, and the Mayo Clinic Brain Bank. Data collection was supported through funding by NIA grants P50 AG016574, R01 AG032990, U01 AG046139, R01 AG018023, U01 AG006576, U01 AG006786, R01 AG025711, R01 AG017216, R01 AG003949, NINDS grant R01 NS080820, CurePSP Foundation, and support from Mayo Foundation. Study data includes samples collected through the Sun Health Research Institute Brain and Body Donation Program of Sun City, Arizona. The Brain and Body Donation Program is supported by the National Institute of Neurological Disorders and Stroke (U24 NS072026 National Brain and Tissue Resource for Parkinson's Disease and Related Disorders), the National Institute on Aging (P30 AG19610 Arizona Alzheimer's Disease Core Center), the Arizona Department of Health Services (contract 211002, Arizona Alzheimer's Research Center), the Arizona Biomedical Research Commission (contracts 4001, 0011, 05-901 and 1001 to the Arizona Parkinson's Disease Consortium) and the Michael J. Fox Foundation for Parkinson's Research.

\section{Authors' contributions}

NRP and TKS conceptualized and designed the study. Analysis and data interpretation were carried out by all authors (TKS, GAP, NRP). TS drafted the manuscript. The authors (TKS, GAP, NRP) have read and approved the final submitted version of the manuscript.

\section{Funding}

Not applicable.

\section{Availability of data and materials}

Data used in this study was obtained from the Synapse data repository (https://www.synapse.org/\#!Synapse:syn5550404). Subsequent analyses not included in the additional materials are available by the author upon request.

\section{Ethics approval and consent to participate}

Access to download the datasets used in this study was approved under the North Texas Regional IRB exempt protocol \#2016-090.

\section{Consent for publication}

Not applicable.

\section{Competing interests}

The authors declare that they have no competing interests.

Received: 19 December 2019 Accepted: 29 April 2020

Published online: 19 May 2020

References

1. Johri A, Beal MF. Mitochondrial dysfunction in neurodegenerative diseases. J Pharmacol Exp Ther. 2012:342(3):619-30.

2. Swerdlow RH, Koppel S, Weidling I, Hayley C, Ji Y, Wilkins HM. Mitochondria, Cybrids, aging, and Alzheimer's disease. Prog Mol Biol Transl Sci. 2017;146: 259-302.

3. Ojala D, Montoya J, Attardi G. tRNA punctuation model of RNA processing in human mitochondria. Nature. 1981;290(5806):470-4.

4. Powell CA, Nicholls TJ, Minczuk M. Nuclear-encoded factors involved in post-transcriptional processing and modification of mitochondrial tRNAs in human disease. Front Genet. 2015;6:79.

5. Duechler M, Leszczyńska G, Sochacka E, Nawrot B. Nucleoside modifications in the regulation of gene expression: focus on tRNA. Cell Mol Life Sci. 2016; 73(16):3075-95.

6. Voigts-Hoffmann F, Hengesbach M, Kobitski AY, van Aerschot A, Herdewijn P, Nienhaus GU, et al. A methyl group controls conformational equilibrium in human mitochondrial tRNALys. J Am Chem Soc. 2007;129(44):13382-3.

7. Helm M, Giegé R, Florentz C. A Watson-Crick Base-pair-disrupting methyl group (m1A9) is sufficient for cloverleaf folding of human mitochondrial tRNALys. Biochemistry. 1999:38(40):13338-46.

8. Zhang C, Jia G. Reversible RNA modification N(1)-methyladenosine (m(1)a) in mRNA and tRNA. Genomics Proteomics Bioinformatics. 2018;16(3):155-61.

9. Bohnsack MT, Sloan KE. The mitochondrial epitranscriptome: the roles of RNA modifications in mitochondrial translation and human disease. Cell Mol Life Sci. 2018;75(2):241-60.

10. Lopez Sanchez MIG, Mercer TR, Davies SMK, Shearwood A-MJ, Nygård KKA, Richman TR, et al. RNA processing in human mitochondria. Cell Cycle. 2011; 10(17):2904-16.

11. Ovcharenko A, Rentmeister A. Emerging approaches for detection of methylation sites in RNA. Open Biol. 2018;8(9):180121.

12. Schwartz S, Motorin Y. Next-generation sequencing technologies for detection of modified nucleotides in RNAs. RNA Biol. 2016;14(9):1124-37.

13. Mercer TR, Neph S, Dinger ME, Crawford J, Smith MA, Shearwood A-MJ, et al. The human mitochondrial transcriptome. Cell. 2011;146(4):645-58.

14. Hauenschild R, Tserovski L, Schmid K, Thüring K, Winz M-L, Sharma S, et al. The reverse transcription signature of $\mathrm{N}$-1-methyladenosine in RNA-Seq is sequence dependent. Nucleic Acids Res. 2015;43(20):9950-64.

15. Hodgkinson A, Idaghdour Y, Gbeha E, Grenier J-C, Hip-Ki E, Bruat V, et al. High-resolution genomic analysis of human mitochondrial RNA sequence variation. Science. 2014;344(6182):413-5.

16. Price AL, Patterson NJ, Plenge RM, Weinblatt ME, Shadick NA, Reich D. Principal components analysis corrects for stratification in genome-wide association studies. Nat Genet. 2006;38:904.

17. Magis AT, Funk CC, Price ND. SNAPR: a bioinformatics pipeline for efficient and accurate RNA-seq alignment and analysis. IEEE Life Sci Lett. 2015;1(2): 22-5.

18. Patterson N, Price AL, Reich D. Population structure and Eigenanalysis. PLoS Genet. 2006;2(12):e190.

19. Purcell S, Neale B, Todd-Brown K, Thomas L, Ferreira Manuel AR, Bender D, et al. PLINK: a tool set for whole-genome association and population-based linkage analyses. Am J Hum Genet. 2007:81(3):559-75.

20. Watanabe K, Taskesen E, van Bochoven A, Posthuma D. Functional mapping and annotation of genetic associations with FUMA. Nat Commun. 2017:8(1): 1826. 
21. de Leeuw CA, Mooij JM, Heskes T, Posthuma D. MAGMA: generalized geneset analysis of GWAS data. PLoS Comput Biol. 2015;11(4):e1004219.

22. Metsalu T, Vilo J. ClustVis: a web tool for visualizing clustering of multivariate data using principal component analysis and heatmap. Nucleic Acids Res. 2015;43(W1):W566-W70.

23. Muruganujan $A$, Ebert $D, M i H$, Thomas PD, Huang X. PANTHER version 14: more genomes, a new PANTHER GO-slim and improvements in enrichment analysis tools. Nucleic Acids Res. 2018;47(D1):D419-D26.

24. Swerdlow RH. Mitochondria and mitochondrial cascades in Alzheimer's disease. J Alzheimers Dis. 2018;62(3):1403-16.

25. Albers DS, Swerdlow RH, Manfredi G, Gajewski C, Yang L, Parker WD, et al. Further evidence for mitochondrial dysfunction in progressive Supranuclear palsy. Exp Neurol. 2001;168(1):196-8.

26. Reas ET. Amyloid and tau pathology in Normal cognitive aging. J Neurosci. 2017:37(32):7561.

27. Tufi R, Gandhi S, de Castro IP, Lehmann S, Angelova PR, Dinsdale D, et al. Enhancing nucleotide metabolism protects against mitochondrial dysfunction and neurodegeneration in a PINK1 model of Parkinson's disease. Nat Cell Biol. 2014;16(2):157-66.

28. Chen Y, Neve RL, Liu H. Neddylation dysfunction in Alzheimer's disease. J Cell Mol Med. 2012;16(11):2583-91.

29. Aguilar BJ, Zhu Y, Lu Q. Rho GTPases as therapeutic targets in Alzheimer's disease. Alzheimers Res Ther. 2017;9(1):97.

30. Fransson A, Ruusala A, Aspenström P. Atypical rho GTPases have roles in mitochondrial homeostasis and apoptosis. J Biol Chem. 2003;278(8):6495502.

31. Saheki Y, De Camilli P. Endoplasmic reticulum-plasma membrane contact sites. Annu Rev Biochem. 2017:86(1):659-84.

32. Traaseth N, Elfering S, Solien J, Haynes V, Giulivi C. Role of calcium signaling in the activation of mitochondrial nitric oxide synthase and citric acid cycle. Biochimica et Biophysica Acta (BBA) - Bioenergetics. 2004;1658(1):64-71.

33. Burgoyne T, Patel S, Eden ER. Calcium signaling at ER membrane contact sites. Biochimica et Biophysica Acta (BBA) - Mol Cell Res. 2015:1853(9):2012-7.

34. Chapard C, Meraldi P, Gleich T, Bachmann D, Hohl D, Huber M. TRAIP is a regulator of the spindle assembly checkpoint. J Cell Sci. 2014:127(24):5149.

35. Khurana V, Merlo P, DuBoff B, Fulga TA, Sharp KA, Campbell SD, et al. A neuroprotective role for the DNA damage checkpoint in tauopathy. Aging Cell. 2012;11(2):360-2.

36. Ghoshal S, Tyagi R, Zhu Q, Chakraborty A. Inositol hexakisphosphate kinase1 interacts with perilipin1 to modulate lipolysis. Int J Biochem Cell Biol. 2016;78:149-55.

37. Zhu Q, Ghoshal S, Rodrigues A, Gao S, Asterian A, Kamenecka TM, et al. Adipocyte-specific deletion of Ip6k1 reduces diet-induced obesity by enhancing AMPK-mediated thermogenesis. J Clin Invest. 2016;126(11):427388.

38. Crocco P, Saiardi A, Wilson MS, Maletta R, Bruni AC, Passarino G, et al. Contribution of polymorphic variation of inositol hexakisphosphate kinase 3 (IP6K3) gene promoter to the susceptibility to late onset Alzheimer's disease. Biochim Biophys Acta (BBA) - Mol Basis Dis. 2016;1862(9):1766-73.

39. Szijgyarto Z, Garedew A, Azevedo C, Saiardi A. Influence of inositol pyrophosphates on cellular energy dynamics. Science. 2011;334(6057):802.

40. Wan Z, Mah D, Simtchouk S, Kluftinger A, Little JP. Role of amyloid $\beta$ in the induction of lipolysis and secretion of adipokines from human adipose tissue. Adipocyte. 2014;4(3):212-6.

41. Schrauwen P, Schrauwen-Hinderling V, Hoeks J, Hesselink MKC. Mitochondrial dysfunction and lipotoxicity. Biochimica et Biophysica Acta (BBA) - Mol Cell Biol Lipids. 2010;1801(3):266-71.

42. Rentzsch P, Witten D, Cooper GM, Shendure J, Kircher M. CADD: predicting the deleteriousness of variants throughout the human genome. Nucleic Acids Res. 2018;47(D1):D886-D94.

43. Ali AT, Idaghdour Y, Hodgkinson A. Nuclear genetic regulation of human mitochondrial RNA modification. bioRxiv. 2019;666339. https://doi.org/10. 1101/666339.

44. Idaghdour Y, Hodgkinson A. Integrated genomic analysis of mitochondrial RNA processing in human cancers. Genome Med. 2017;9(1):36.

\section{Publisher's Note}

Springer Nature remains neutral with regard to jurisdictional claims in published maps and institutional affiliations.

Ready to submit your research? Choose BMC and benefit from:

- fast, convenient online submission

- thorough peer review by experienced researchers in your field

- rapid publication on acceptance

- support for research data, including large and complex data types

- gold Open Access which fosters wider collaboration and increased citations

- maximum visibility for your research: over $100 \mathrm{M}$ website views per year

At BMC, research is always in progress.

Learn more biomedcentral.com/submissions 